\title{
NBS: A Community-Based Approach to Developing an Integrated Surveillance System
}

\author{
Christi Hildebrandt ${ }^{\star}$, Jennifer Ward and Akshar Patel \\ CSRA, Chattanooga, TN, USA
}

\section{Objective}

The NEDSS Base System (NBS) is designed and developed using input from $\mathrm{CDC}$ programs, public health standards organizations, as well as its expansive user community. This community-based approach to development of an integrated surveillance system is described.

\section{Introduction}

The NEDSS Base System (NBS) is a web-based, standardsdriven, integrated disease surveillance system launched in 2001 and is currently in use in twenty-two public health jurisdictions. Over the past fifteen years, the NBS has grown into a highly functional, modern application that supports: case management, electronic data exchange, metadata-driven data collection, workflow decision support, and a host of other functionalities, all of which are defined and designed through a community-based approach.

\section{Methods}

In order to encourage open communication and collaboration across and among the community, there is a well-publicized, longstanding communication plan in place. Further, tools such as an online collaboration and support forum, NBSCentral, are made available to any person who requests access. Also, the NBS source code is provided in an open source package to anyone interested, along with each release, and a demonstration version of the application can be accessed online by anyone to review the latest release of the application. All of these channels are in place to ensure there are ways for all who have in interest in collaborating to easily participate.

The NBS community regularly meets to provide input into further development of the system, as well as discuss topics affecting public health. As a community, members:

- Share best practices, tools, and lessons learned across jurisdictions

- Share innovative local approaches to disease surveillance and reporting

- Access NBSCentral for support and collaboration

- Participate in the change control and planning process for each NBS release

- Work collaboratively with CDC to define high-level vision and priorities

- Provide input to create community-defined requirements for system development

- Participate in weekly subject matter expert (SME) calls to discuss development and best practices

- Have the opportunity to participate in beta testing for releases

- Attend a bi-weekly NBS User Group (NUG) call to discuss the system as well as reach out to colleagues to brainstorm creative solutions to common problems in public health surveillance

All meetings with stakeholders are recorded and shared with the larger community to ensure full transparency and for historical reference.

\section{Results}

Through this inclusive development approach, the NBS has evolved into a highly extensible, configurable system that can meet that needs of twenty-two very different public health jurisdictions; the system can be implemented without the need for custom development in a relatively short timeframe due to the fact that it was designed to meet the needs of many. Further, it has encouraged interoperability projects, such as: piloting electronic case reporting use cases between NBS implementation sites and building and sharing electronic case investigation forms for data collection using the NBS Page Builder module. All NBS sites use the same translation routes for electronic lab report, case report, and Nationally Notifiable Disease message processing - embracing the build once, use many concept. Most recently, having this collaboration network in place made it very easy for the NBS community to quickly adapt to the changing needs of Zika virus surveillance.

\section{Conclusions}

It does require clear definition of processes and communication channels, as well as regular update and transparency into the process for community-based development to work. However, when the proper tools and processes are in place, the benefits of collaboration with all key stakeholders are exponential when realized. Developing an application in this way has provided NBS users not only with a much better, integrated surveillance system, but also a forum for understanding how other jurisdictions have solved similar issues; it provides a springboard for sharing and building upon novel ideas and new approaches in public health surveillance.

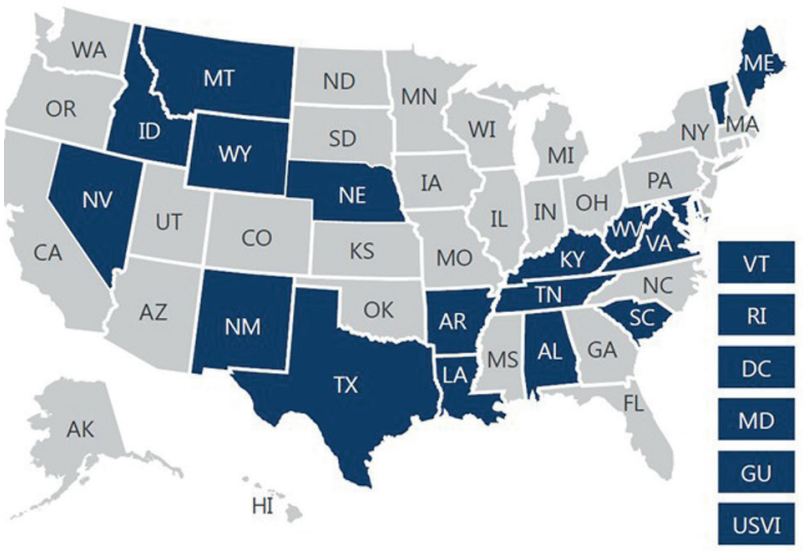

Keywords

NBS; Surveillance; Community; Integrated; collaborative

\section{Acknowledgments}

The NBS is funded by the Centers for Disease Control and Prevention and is supported by the NBS User Group (NUG), which consists of state, local and territorial public health staff and their representatives.

\section{${ }^{*}$ Christi Hildebrandt}

E-mail: christi.hildebrandt@csra.com 